\title{
A case of intraplacental gestational choriocarcinoma; characterised by the methylation pattern of the early placenta and an absence of driver mutations
}

\author{
Philip Savage ${ }^{1,2^{*}}$ (D), David Monk ${ }^{3}$, Jose R. Hernandez Mora ${ }^{3}$, Nick van der Westhuizen², Jennifer Rauw², \\ Anna Tinker ${ }^{2}$, Wendy Robinson ${ }^{4}$, Qianqian Song ${ }^{5}$, Michael J. Seckl ${ }^{1}$ and Rosemary A. Fisher ${ }^{1,6}$
}

\begin{abstract}
Background: Gestational choriocarcinoma is a rare malignancy believed to arise from the trophoblast cells of the placenta. Despite the frequently aggressive clinical nature, choriocarcinoma has been routinely curable with cytotoxic chemotherapy for over 50 years. To date little is known regarding the route to oncogenesis in this malignancy.

Methods: In a case of intraplacental choriocarcinoma, we have performed detailed genetic studies including microsatellite analysis, whole genome sequencing (WGS) and methylation analysis of the tumour and surrounding mature placenta.

Results: The results of the WGS sequencing indicated a very low level of mutation and the absence of any driver mutations or oncogene activity in the tumour. The methylation analysis identified a distinctly different profile in the tumour from that of the mature placenta. Comparison with a panel of reference methylation profiles from different stages of placental development indicated that the tumour segregated with the first trimester samples.

Conclusions: These findings suggest that gestational choriocarcinoma is likely to arise as a result of aberrations of methylation during development, rather than from DNA mutations.

The results support the hypothesis that gestational choriocarcinoma arises from a normally transient early trophoblast cell. At this point in development this cell naturally has a phenotype of rapid division, tissue invasion and sensitivity to DNA damaging chemotherapy that is very similar to that of the mature choriocarcinoma cell.
\end{abstract}

Keywords: Oncogenesis, Trophoblast, Placenta, Choriocarcinoma, Methylation, Epigenetics, Pregnancy, Chemotherapy

\section{Background}

Gestational Trophoblastic Neoplasia (GTN) are a group of rare conditions that arise from the cells of conception. The most frequent forms are the pre-malignant genetically abnormal complete and partial hydatidiform moles which arise from an androgenetic

\footnotetext{
* Correspondence: savage13561@msn.com

${ }^{1}$ Trophoblastic Tumour Screening \& Treatment Centre, Charing Cross Hospital Campus of Imperial College, London, UK

${ }^{2} \mathrm{BCCA}$, Victoria, BC, Canada

Full list of author information is available at the end of the article
}

or triploid dispermic conceptus respectively. The clinically more complex malignant diagnoses of gestational choriocarcinoma, placental site trophoblastic tumour (PSTT) and epithelioid trophoblastic tumour (ETT), whilst on occasion can arise from a molar pregnancy, more usually each arise from pregnancies that have the normal genetic complement [1].

Clinically gestational choriocarcinoma is frequently characterised as an invasive, fast growing and aggressive cancer. Presentation during the causative pregnancy is rare and most cases are identified some months or years following

(c) The Author(s). 2019 Open Access This article is distributed under the terms of the Creative Commons Attribution 4.0 International License (http://creativecommons.org/licenses/by/4.0/), which permits unrestricted use, distribution, and 
the causative pregnancy often as a result of symptoms from distant metastatic spread. The overall incidence of gestational choriocarcinoma is estimated at 1 case per 50,000 pregnancies and aside from increasing maternal age does not appear to have any other significant risk factors [2].

Despite this rarity and the rapidity of cell growth, gestational choriocarcinoma has been curable with cytotoxic chemotherapy since the 1950 s and in modern series the overall cure rate now approaches 95\% [3, 4].

Conventionally malignancies characteristically arise from previously normal cells that develop the malignant phenotype, including uncontrolled growth and invasion, as a result of a number of DNA mutations [5]. The normally transient early trophoblast cells have a phenotype, including rapid growth, local invasion and stimulation of angiogenesis that is similar to that of a malignant cell. As a result, it has been suggested that gestational choriocarcinoma may not necessarily represent a classical mutation based malignant transformation. Instead these tumours could arise due to the persistence of early trophoblast cells, which have failed to either mature or undergo apoptosis [6].

In contrast to most common malignancies, gestational choriocarcinoma is frequently managed based on a clinical diagnosis without a biopsy and therefore tumour samples of sufficient quantity to permit detailed genetic or methylation analysis are exceptionally rare. As a result, no previous whole genome sequencing or detailed methylation studies have been reported for this rare diagnosis.

Gestational choriocarcinoma is thought to originate from the cytotrophoblast and syncytiotrophoblast cells that develop into the placenta. Since most cases are not diagnosed until months or years after the end of the pregnancy, in the large majority of cases of choriocarcinoma the placenta has been discarded before the diagnosis is clinically apparent. However, in very rare cases an intra-placental choriocarcinoma is noted within the placenta at delivery or on pathological examination. This is therefore the earliest clinical time point for diagnosis of a choriocarcinoma and such material provides a unique opportunity to study the genetic or epigenetic changes that could drive the malignant behaviour of this tumour prior to any additional later acquired changes. Interestingly, even in these 'early' choriocarcinomas approximately $50 \%$ are already associated with metastatic spread. Fortunately, with modern chemotherapy the cure rate for women with these rare intraplacental choriocarcinomas approaches 100\% [7].

Here we have investigated the potential contribution of genetic and epigenetic changes to the pathogenesis of this rare malignancy, using whole genome sequencing and methylation analysis from a recent case of intraplacental gestational choriocarcinoma.

\section{Case presentation}

After an uneventful second pregnancy a 41-year-old caucasian woman delivered a 39-week female baby via emergency caesarean section for fetal distress. At delivery the baby was unwell and despite resuscitation, sadly died $19 \mathrm{~h}$ post-delivery.

Macroscopic examination of the placenta demonstrated a $4 \mathrm{~cm}$ abnormality (Fig. 1a). On histopathological review the diagnosis of gestational choriocarcinoma was made based on the morphological appearance of sheets of atypical mononucleated cytotrophoblast admixed with multinucleated syncytiotrophoblast with associated haemorrhage and necrosis (Fig. 1b). A fetal post mortem was not performed.

In response to the diagnosis of choriocarcinoma, the patient was assessed for the presence of metastatic disease with an MRI scan of the head and pelvis and a CT of the chest and abdomen and serial serum human chorionic gonadotrophin (hCG) monitoring. The imaging showed no evidence of distant disease. The hCG level immediately post-partum was 202,499 IU/L and then fell sequentially with the expected 1-2 day half-life

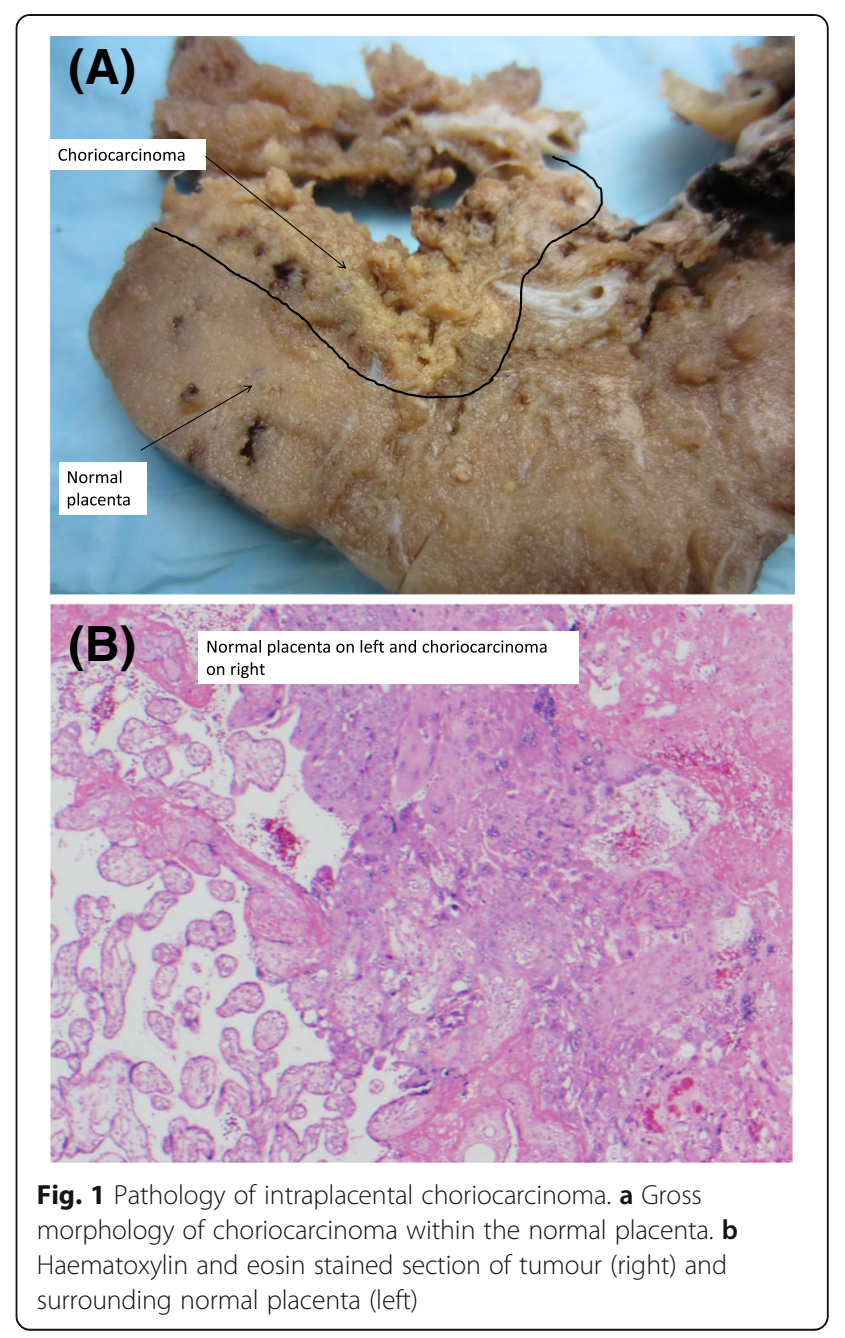


for complete removal of tumour reaching the normal range approximately 30 days post-delivery. On follow up the patient has remained well, with a normal serum hCG for more than 3 years and the chance of relapse is now remote.

\section{Methods}

\section{Genetic investigations}

With the patient's consent and in keeping with the institutional ethics policy further genetic analysis of the placenta and tumour were performed.

\section{Preparation of genomic DNA}

With reference to a consecutive haematoxylin and eosin stained section, tumour tissue and surrounding normal placenta were micro-dissected independently from unstained formalin-fixed paraffin-embedded (FFPE) sections. DNA was prepared from dissected tissue using a QIAmp DNA FFPE Tissue Kit (Qiagen, UK) and the DNA quantified using a Picogreen dsDNA quantitation kit (Life Technologies, UK).

\section{Fluorescent microsatellite genotyping}

To exclude the possibility that the tumour could have been either a metastasis from an occult cancer, a gestational choriocarcinoma from previous pregnancy or have arisen from a concurrent hydatidiform mole genotyping of DNA from the tissue and normal surrounding placenta was performed [8]. Briefly, 5 ng of DNA from the normal placenta and tumour tissue were amplified with a panel of primers for 15 short tandem repeat (STR) loci on 13 autosomes and the amelogenin locus using an AmpFlSTR Identifiler Plus kit (Applied Biosystems, Warrington, UK). PCR products were resolved by capillary electrophoresis using an ABI 3100 Genetic Analyser and genotypes determined using GeneMapper version 5.0 software (Applied Biosystems).

\section{Whole genome sequencing}

Genomic DNA libraries were prepared following Illumina's (Illumina, San Diego, CA) suggested protocol and sequenced by Hiseq X Ten (Illumina) with 150PE. Somatic variants were identified using GATK Best Practices Pipeline. After Illumina sequencing, all produced FASTQ reads were quality-checked and trimmed with FastQC (version 0.11.2 http://www.bioinformatics.babraham.ac.uk/projects/fastqc/) and Trimmomatic (version 0.33). The average coverage of each base in the genome was 36.31 for the tumours and 23.11 for the placenta. The Q30 (\%) was $91.20 \%$. Sequencing reads were aligned to human genome hg19 with the BWA MEM software for both tumour and normal samples. PCR duplications of each BAM file were marked with Picard software (version $1.103 \mathrm{https} / /$ /broadinstitute.github.io/picard/). The BAM files were locally realigned, and the base quality scores were recalibrated with GATK (version 3.1). Single nucleotide variants and Indels were detected using MuTect (version 1.1.6) and Strelka (version 1.0.14) respectively. All the somatic variants were validated with Integrative Genomics Viewer and annotated with Variant Effect Predictor (version 83).

\section{Methylation array hybridization}

FFPE-derived DNA from both the tumour and placenta tissue were hybridized to the Illumina Infinium Methylation EPIC (EPIC) arrays. Bisulphite conversion was performed according to the manufacturer's recommendations for the Illumina Infinium Assay (EZ DNA methylation kit, ZYMO, Orange, CA) and subjected to the Infinium FFPE QC and restoration kit prior to hybridisation.

\section{Data filtering and analysis of methylation signals}

Before analysing the data, possible sources of technical biases that could influence results were excluded. We applied signal background subtraction using default control probes in BeadStudio (version 2011.1_Infinium HD). We discarded probes with a detection $P$-value $>0.01$, containing single nucleotide polymorphisms (SNPs) within the interrogation or extension base as well as those with potential cross-reaction due to multiple sequence homologies. We also excluded probes that lacked signal values in one or more of the DNA samples analysed and that mapped to the X \& Y chromosomes. In total 772,399 probes were investigated. For the analysis of known imprinted ubiquitous differentially methylated regions (DMRs), probes mapping to the intervals identified by Hernandez-Mora et al. were directly examined [9]. The names of each region are in accordance with the recently recommended nomenclature for clinical reporting of imprinted methylation profiles [10]. For placenta-specific imprinted DMRs, only probes mapping to regions with confirmed allelic methylation were interrogated [11]. The common regions of $\mathrm{CpG}$ island Methylator Phenotype (CIMP) were taken from MartinTrujillo et al. [12]. In-house bioinformatics $\mathrm{R}$ scripts were utilized for statistics to identify loci with different methylation profiles between the two samples and to compare the FFPE-derived DNA methylation profiles with those obtained from high-molecular weight DNAs extracted directly from paired first trimester chorionic villous samples and term biopsies (GEO repository GSE121056) [13]. The data from the FFPE placenta and tumour are available in the GEO repository with the accession number GSE125386.

\section{Bisulphite methylation analyses}

For confirmation PCR analyses, loci with the greatest significant difference identified using the EPIC arrays were targeted using standard bisulphite PCR. Furthermore, these and those analysed by pyrosequencing, had underlying 
converted sequences compatible with optimal primer design resulting in short products incorporating numerous CpG dinucleotides to ensure efficient amplification in FFPE-degraded input DNA.

Allelic PCR: Approximately $1 \mu \mathrm{g}$ FFPE-derived DNA was converted using the EZ DNA Methylation-Gold kit (Zymo) following manufacturer's instructions for short incubation times to avoid additional fragmentation of the DNA during the treatment. Approximately $5 \mu \mathrm{l}$ of bisulphite converted DNA was used in each amplification reaction using Immolase Taq polymerase (Bioline, UK) for 45 cycles and the resulting PCR product sub-cloned into pGEM-T easy vector (Promega) for sequencing (for primer sequences see Additional file 1: Table S1).

Pyrosequencing: Standard bisulphite PCR was used to amplify $50 \mathrm{ng}$ of bisulphite converted DNA with the exception that one primer was biotinylated (for primer sequence see Additional file 1: Table S1). The entire biotinylated PCR product (diluted to $40 \mu \mathrm{l}$ ) was mixed with $38 \mu \mathrm{l}$ of Binding buffer and $2 \mu \mathrm{l}(10 \mathrm{mg} / \mathrm{ml})$ streptavidin-coated polystyrene beads. After incubation at $65^{\circ} \mathrm{C}$, DNA was denatured with $50 \mu \mathrm{l} 0.5 \mathrm{M} \mathrm{NaOH}$. The single-stranded DNA was hybridized to $40-\mathrm{pmol}$ sequencing primers dissolved in $11 \mu \mathrm{l}$ annealing buffer at $90^{\circ} \mathrm{C}$. For sequencing, a primer was designed to the opposite strand to the biotinylated primer used in the PCR reaction. The pyrosequencing reaction was carried out on a PyroMark Q96 instrument. The peak heights were determined using Pyro Q-CpG1.0.9 software (Biotage, Sweden).

\section{Results}

\section{Fluorescent microsatellite genotyping analysis}

The fluorescent microsatellite genotyping analysis (Additional file 2: Table S2) demonstrated the tumour to have the same genotype as the healthy placenta, confirming the identity of the tumour as a non-molar gestational choriocarcinoma arising in the current pregnancy. Furthermore, these results were endorsed by analysis of 65 highly informative SNPs present on the Illumina EPIC methylation array.

\section{Whole genome sequencing}

Whole genome sequencing did not demonstrate any mutations of the common cancer associated oncogenes or any other driver mutations. The overall mutational load was extremely low, both in the number of detected mutations and also the proportion of the DNA prepared from the malignant cells that carry any of these apparent mutations (Table 1). Overall the results indicate that within this tumour the mutational load was extremely low and unlikely to be of biological significance.

\section{Methylation studies}

In contrast the epigenetic studies demonstrated that the pattern of methylation is markedly different between the tumour, the surrounding normal placenta or reference unrelated term placental samples. To determine whether there was any resemblance of the methylation profile in the tumour with early placental samples we obtained DNA from 12-week chorionic villus biopsies. Interestingly, using both partitioning and hierarchical clustering, we observed that the tumour has a genome-wide methylation profile resembling the first trimester chorionic villous samples, whilst the placenta is located in a separate branch with normal term controls (Fig. 2a). To identify specific loci that are differentially methylated between the tumour and placenta we performed an unsupervised search between the two samples. This revealed 177816 hypermethylated positions and 405383 hypomethylated by $-1+2.5 \%(0.025 ß)$, the accepted detection resolution of the Infinium assays [14]. Of these 336 differed by more than $50 \%$ (0.5ß) (Fig. 2b). Subsequently we performed bumphunter analysis to identify multiple probe clusters of which 2645 were hypermethylated and 3365 hypomethylated (Additional file 3: Table S3). In general, hypermethylated intervals were larger (top 200 candidates; mean length $877 \mathrm{bp}$ SD $594 \mathrm{bp}$, containing on average 10.4 probes) than hypomethylated regions (top 200 candidates; mean length $257 \mathrm{bp}$ SD $325 \mathrm{bp}$ containing on average 3.5 probes), however these differences may reflect a bias in assay design since probes in intergenic regions are underrepresented. When the genomic location of the differentially methylated probes is taken into consideration, "CpG island" probes are clearly less prone to be hypomethylated in the tumour with respect to the placenta, while "CpG islands, shelf and shores" are hypermethylated (Fig. 2c). To validate the methylation profiles obtained from the EPIC array comparisons we performed bisulphite PCR and sub-cloning of two regions. In each case we confirm the profile observed, with TLL1 and ZNF350 being more methylated in the tumour than placenta (Fig. 2d).

Next, we analysed the methylation profiles at imprinted differentially methylated regions (DMRs) since these are closely linked to both placental development and tumourigenesis [15]. Of the $40 \mathrm{ubi}$ quitous imprinted DMRs present on the EPIC platform loss-of-methylation (LOM) was far more frequent than gains-of-methylation (GOM) with 18 regions comparable between the two samples, 16 modestly hypomethylated and 6 hypermethylated in the tumour (Fig. 2e, Additional file 4: Table S4). One region of GOM, at the paternally-methylated $Z D B F 2$ DMR is as a direct consequence of hypomethylation of the adjacent maternally-methylated GPR1-AS1 DMR which is responsible for appropriate allelic 


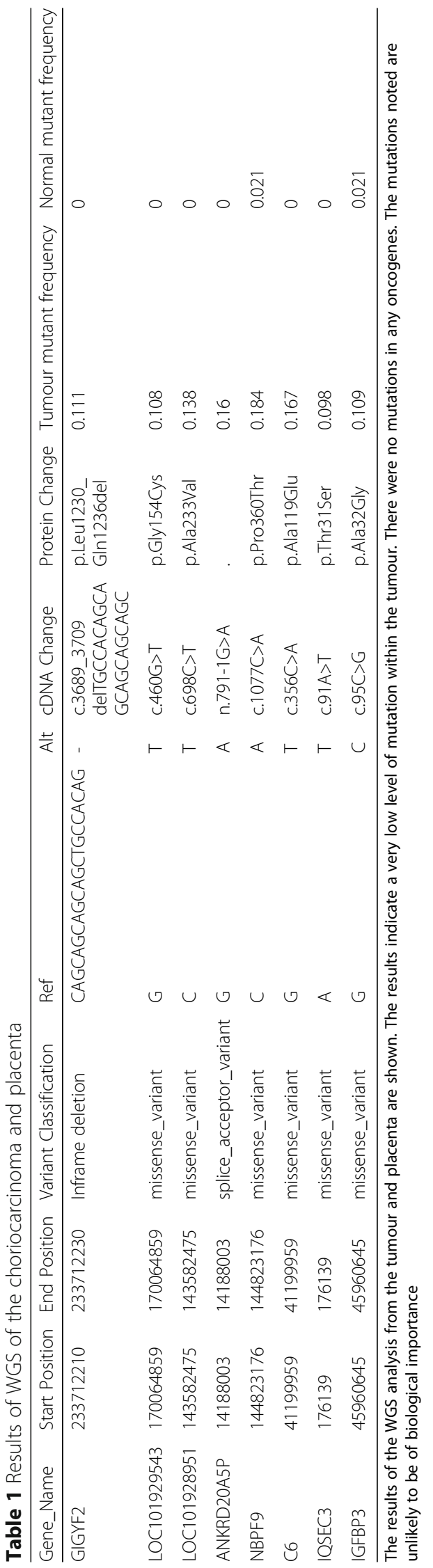


(A)
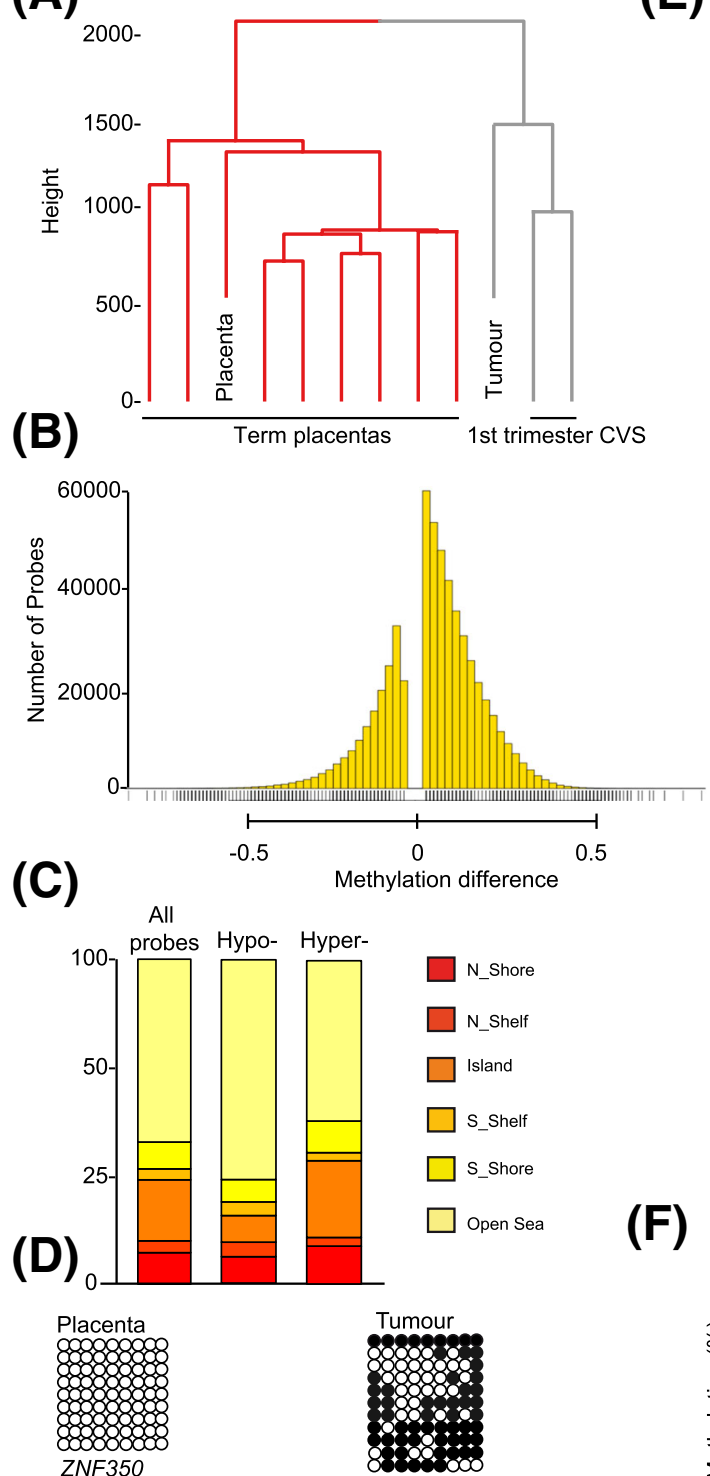

ZNF350
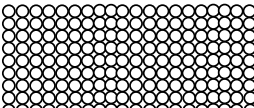

888888
(E)

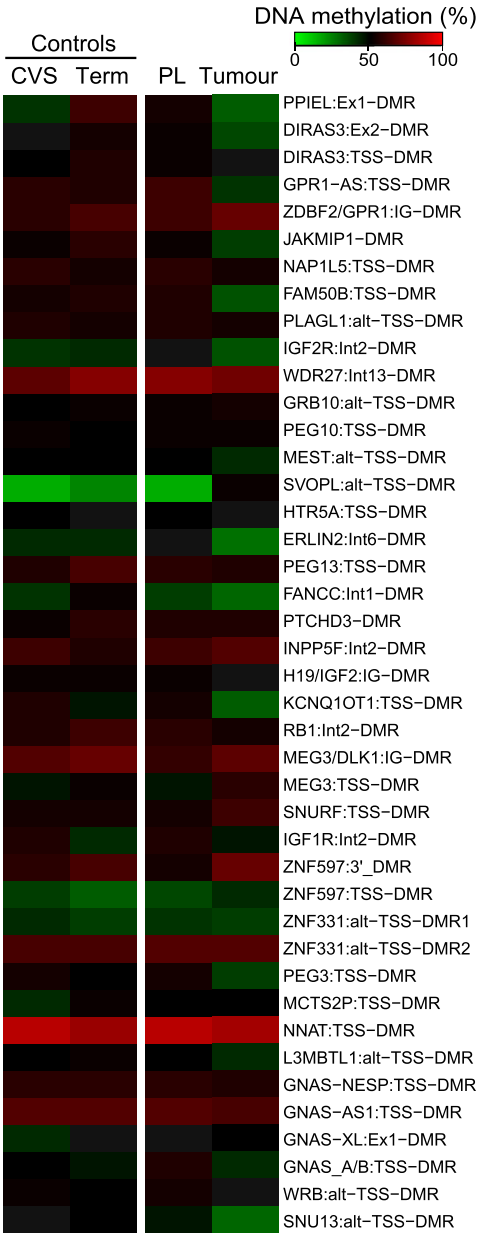

(F)

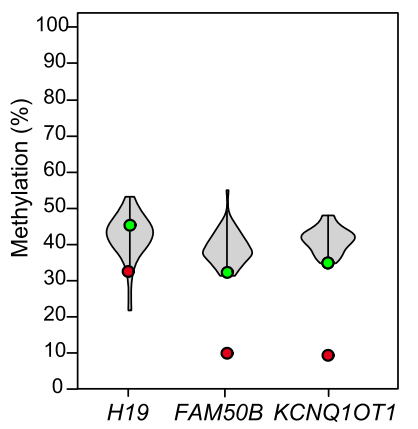

Fig. 2 Characterization of DNA methylation in the placenta and tumour using the Illumina EPIC methylation array. a Hierarchical clustering of global methylation for placenta-tumour paired samples with 8 term placenta and 2 first trimester CVS samples. b Bar graph of the distribution of the hypo- (left side) and hypermethylated (right side) probes with a difference greater that $-1+2.5 \%(0.025 ß)$ when comparing the tumour and paired placenta. c Classification of probes with differential methylation according to genomic location. The bar chart illustrates probe enrichment classified by Illumina Infinium annotation. d Bisulphite confirmation of methylation difference between tumour and paired placenta samples. Each circle represents a single CpG dinucleotide on a DNA strand. (•) Methylated cytosine, (o) unmethylated cytosine. Each row corresponds to an individual cloned sequence. e Heatmap of the ubiquitous imprinted DMR probes in control first trimester CVS and term placenta biopsies as well as the tumour and paired placenta samples. The values represent the average of all probes mapping to each region. $\mathbf{f}$ Methylation levels of ubiquitous imprinted DMRs in the placenta (green dot), tumour (red dot) and controls (violin plots $n=16$ ) quantified by pyrosequencing

methylation at this domain in a hierarchical manner [16]. To ensure the EPIC array data truly reflects the methylation profile at imprinted DMRs, we performed pyrosequencing for the H19, KCNQ1OT1, and $F A M 50 B$ regions which confirmed that the tumour sample is $22-24 \%$ less methylated than the 
corresponding placenta (Fig. 2f). We subsequently extended this analysis to placenta-specific imprinted DMRs. Of the samples with appropriate methylation in the placenta samples (these are polymorphic epialleles so we only analysed those with a pattern consistent with allelic methylation in the normal placenta sample), 97 regions were comparable between the two samples and 69 modestly hypomethylated (Additional file 5: Table S5), with the levels at the CMTM3 and GLIS3 DMRs confirmed by pyrosequencing (Additional file 7: Figure S1A and B).

Finally, potential changes in methylation associated with the tumourigenic process have previously been reported in samples with global hypomethylation. Widespread CpG island promoter hypermethylation, also referred to as $\mathrm{CpG}$ island methylator phenotype (CIMP), has been reported for many tumour types [17]. With the exception of CRABP1, the analysis of 23 CIMP regions revealed very little tumour-associated hypermethylation (Additional file 7: Figure S1C, Additional file 6: Table S6) suggesting that CIMP may not be a universal phenomenon in gestational choriocarcinomas.

\section{Discussion}

To date there has been relatively little work examining the potential role of genetic changes in the pathogenesis of gestational tumours. However, recent data from targeted sequencing of 6 cases of gestational choriocarcinoma, examining 637 cancer related genes, has indicated an extremely low level of somatic mutation [18]. Similarly targeted sequencing of cases of the PSTT and ETT, gestational malignancies that arise slightly later in placental development, have also demonstrated very low levels of mutation and a lack of any repetitive or driver mutations [19].

Intra-placental gestational choriocarcinomas, in which the tumour is identified in the placenta during pregnancy or at delivery are extremely rare but represent a very early form of gestational malignancy [7]. As a result, any genetic changes identified in an intra-placental gestational choriocarcinoma are likely to represent the earliest changes associated with tumour development.

In this report, we have aimed to more fully characterise the genetic and epigenetic changes in a case of intra-placental gestational choriocarcinoma. We first confirmed the gestational origin of the malignancy found within the placenta by microsatellite genotyping analysis, the results shown in Additional file 2: Table S2 demonstrate identical results at all 16 loci. This result excludes the possibility that the tumour in the placenta could either have been a metastasis from an occult primary cancer or have arisen from a prior conception or concurrent molar pregnancy.
Further analysis by whole genome sequencing of the tumour with comparison to the neighbouring healthy placenta failed to demonstrate any mutations in any established oncogenes or any appreciable mutations elsewhere in the genome as shown in Table 1 . This finding, a lack of any significant mutation in this case, supports the earlier genetically bland findings obtained from sequencing of a limited number of genes in a panel of trophoblast tumours $[18,19]$. A number of paediatric malignancies, which each share a relatively short oncogenesis timeframe, similarly have very low overall levels of mutations. In these other rare diagnoses, the cells gain their malignant phenotype from single driver mutations in the case of infantile acute lymphoblastic leukaemia and paediatric rhabdoid tumours or epigenetic changes alone in CIMP-positive ependymoma [20-22].

It is apparent that the time frame for the development of malignancy in gestational choriocarcinoma is even shorter than for these paediatric malignancies. We have previously questioned whether accumulation of mutations, as in the common epithelial malignancies, could be the route to oncogenesis of this rare malignancy [6]. The demonstration of a lack of significant mutation in this case analysed by WGS and in other cases of trophoblast tumours previously analysed by limited genomic profiling would support the argument that gestational choriocarcinoma does not appear to be a mutation driven malignancy $[18,19]$.

Normal early trophoblast cells appear to share many characteristics of malignant choriocarcinoma cells including rapid proliferation, hCG production, an ability to invade into other tissues, stimulation of angiogenesis and also the extreme sensitivity to DNA damaging chemotherapy [23, 24]. As a result, it has been hypothesised that gestational choriocarcinoma may occur not as a result of genetic change but via the inappropriate persistence of normally transient primitive trophoblast cells, that are unable to either mature or undergo apoptosis. As a result, these cells could be locked in a frozen developmental state of an early trophoblast cell's and retain much of that cells normal phenotypically malignant phenotype [6].

In the absence of a mutational cause for oncogenesis we examined the methylation status of the tumour DNA. Normal placental development is associated with wide spread epigenetic changes, the transition from first to third trimester being associated with increasing hypermethylation [25-27]. Examination of the methylation profile using high-throughput arrays of the tumour and normal placenta in the current case showed the methylation patterns of the tumour and the mature placenta differ significantly (Fig. 2). The tumour sample was hypomethylated compared to the placenta at most ubiquitous and placenta imprinted DMRs. Despite the 
differences detected using EPIC methylation arrays being modest, validation using quantitative pyrosequencing revealed a similar degree of hypomethylation at three ubiquitous and two placenta specific imprinted DMRs. Hierarchical clustering demonstrated the tumour to cluster with first trimester chorionic villous samples while the placenta clustered with the term controls. This demonstration that the methylation profile of the tumour is close to that of a first trimester placenta rather than a mature placenta supports the hypothesis that gestational choriocarcinoma arises from an early trophoblast cell [23].

The biological processes involved in early pregnancy share several phenotypic hallmarks of cancer. After undergoing epithelial-to-mesenchymal transition, placental cells invade and migrate within the endometrium whilst evading maternal immune system. The similarities between placenta development and cancer also extends to their unusual epigenomes. Recently, Nordor and colleagues reported that loci undergoing widespread hypomethylation in placenta were of similar size and location as those distinguishing tumours from matched normal tissues [28].

In addition to the widespread intergenic hypomethylation evident in this case of intra-placental gestational choriocarcinoma, many promoter $\mathrm{CpG}$ islands underwent hypermethylation. Promoter hypermethylation of tumour-suppressor genes is a frequent and key event in tumorigenesis. Of the promoters gaining methylation in our sample, many have already been shown to have tumour suppressor activity, including RORa, FAN1, PRDM1, CYGB, L3MBTL4, EPB41L3, with ZNF471 and ZNF671 being specifically silenced by methylation $[29,30]$. Strikingly, like ZNF471 and ZNF671, 27 of the top 200 hypermethylated genes identified in this study map to zincfinger genes, with $75 \%$ mapping to the Chr19q41-43 cluster, suggesting that altered expression of these DNAbinding proteins maybe involved in the development of gestational choriocarcinoma.

The concept that the onset of malignancy can cause a halt in the normal developmental pathways and prevent onset of the normal processes of apoptosis is well established in the lymphoid malignancies [31]. The impact of stopping the normal development of the trophoblast cell at the time of transformation to the choriocarcinoma cell, may explain the extreme sensitivity of these malignant cells to DNA damaging chemotherapy drugs. This characteristic of normal early trophoblast cells is exploited in the medical management of ectopic pregnancy which is effectively treated with low doses of the chemotherapy agent methotrexate [32].

The ability of methylation changes alone to result in oncogenesis has already been demonstrated in CIMPpositive ependymoma, a rare malignancy occurring in children. Studies looking at a panel of these tumours have demonstrated that there are only overall very low levels of mutations with no discernable patterns or driver mutations [22]. In this form of ependymoma it appears that the production of the malignant phenotype occurs via epigenetic changes including transcriptional silencing of key developmental genes.

It is difficult to draw firm conclusions based on the genome analysis of FFPE material from a single case of intraplacental gestational choriocarcinoma. However, our data suggest that the malignant phenotype in this diagnosis, as in ependymomas, may be based on epigenetic rather than genetic changes. We predict that the changes at imprinted loci lead to a block in the development of early trophoblast cells at a stage where their phenotype is naturally close to that of a malignant cell. The importance of the differing basic physiology of the cell origin in determining the sensitivity to cytotoxic chemotherapy is clearly demonstrated in these two malignancies that occur without DNA mutations. Whilst gestational choriocarcinoma is extremely sensitive to chemotherapy and highly curable, CIMP ependymoma which is also methylation driven and has no mutations is extremely resistant to chemotherapy and carries a very poor prognosis [33].

\section{Conclusions}

Based on the initial data available from this case it may be possible to suggest an important difference in the route to oncogenesis between gestational choriocarcinoma and other malignancies. Characteristically malignancies gain their malignant phenotype as a result of an aberrant genetic event. In contrast it appears that in gestational choriocarcinoma the cells do not gain the malignant phenotype, but they fail to lose the essentially malignant phenotype of a primitive trophoblast cell as a result of an aberrant event in the normal progression of methylation changes.

The natural history of gestational choriocarcinoma is extremely varied. The interval from pregnancy to presentation can be very short or can extend to greater than 20 years. Similarly, the clinical course can vary from a widely metastatic fast growing and life-threatening cancer to one that has more indolent behaviour presenting with repeated false positive pregnancy tests, but without clinical symptoms. It is likely that this width of clinical behaviour may be matched by differing patterns of methylation and gene expression. Further studies examining the role of methylation changes in other cases of trophoblastic tumours may provide confirmation of these observations and also a more detailed understanding of this route to oncogenesis. 


\section{Additional files}

Additional file 1: Table S1 PCR primers. (XLSX 9 kb)

Additional file 2: Table S2 Genotyping of DNA from placental villi and tumour tissue showing alleles identified at sixteen informative loci. (DOCX $14 \mathrm{~kb})$

Additional file 3: Table S3 List of loci containing multiple Illumina EPIC probes identified by bumphunter analysis. (XLSX $721 \mathrm{~kb}$ )

Additional file 4: Table S4 Methylation profiles of individual probes mapping within the ubiquitous, imprinted DMRs. (XLSX 62 kb)

Additional file 5: Table S5 Methylation profiles of individual probes mapping within placenta specific imprinted DMRs. (XLSX 60 kb)

Additional file 6: Table S6 Methylation profiles of individual probes mapping within CIMP domains. (XLSX 39 kb)

Additional file 7: Figure S1 Methylation profiling specific loci using the Illumina EPIC methylation array. (A) Heatmap of the placenta-specific imprinted DMR probes in the tumour and paired placenta samples. (B) Methylation levels of GLIS3 and CMTM3, two placenta-specific imprinted DMRs, in the placenta (green dot), tumour (red dot) and controls (violin plots $n=16$ ) quantified by pyrosequencing. (C) Heatmap of the CIMP regions in the tumour and paired placenta samples. Notes, for $(A)$ and $(C)$ the array values represent the average of all probes mapping to each region. (PDF $434 \mathrm{~kb}$ )

\section{Abbreviations}

CIMP: CpG island Methylator Phenotype; DMRs: Differentially methylated regions; ETT: Epithelioid trophoblastic tumour; FFPE: Formalin-fixed paraffinembedded; GOM: Gains-of-methylation; hCG: Human chorionic gonadotrophin; LOM: Loss-of-methylation; PST: Placental site trophoblastic tumour; STR: Short tandem repeat; WGS: Whole genome sequencing

\section{Acknowledgements}

The authors are grateful for the encouragement and support from Prof B Vogelstein.

\section{Authors' contributions}

PS devised the study and drafted the manuscript. JR and AT provided the clinical care. NW reported the histopathology. DM and JHM performed the methylation analysis and interpretation. WR performed additional methylation analysis. QS performed the whole genome analysis and interpretation. MJS critically appraised the project and revised the manuscript. RAF prepared DNA, analysed data and contributed to the manuscript writing. All the authors have read and approved the final manuscript.

\section{Funding}

There was no specific funding for this project.

JHM and DM are been funded in part by Ministerio de Economía, Industria y Competitividad (MINECO), which is part of Agencia Estatal de Investigación (AEI), through the projects BFU2014-53093-R and BFU2017-85571-R (Cofunded by European Regional Development Fund; ERDF, a way to build Europe). We thank CERCA Programme / Generalitat de Catalunya for institutional support.

RAF acknowledges the support of the UK Department of Health and the National Institute for Health Research (NIHR) Imperial Biomedical Research Centre for funding for the DNA preparation and microsatellite genotyping. MJS acknowledges support from the Imperial National Institute of Health Research (NIHR) Biomedical Research Centre and NIHR/ Cancer Research UK Experimental Cancer Medicine Centre.

\section{Availability of data and materials}

The DNA sequencing data is available on request.

The methylation data has been uploaded to the GEO repository as detailed in the text.

\section{Ethics approval and consent to participate}

This study was approved by the institutional review board of the University of British Columbia. Children's and Women's Research Ethics Board Approval number: H12-00145.

\section{Consent for publication}

Written informed consent was obtained from the patient for publication of this project.

\section{Competing interests}

The authors declare that they have no competing interests.

\section{Author details}

${ }^{1}$ Trophoblastic Tumour Screening \& Treatment Centre, Charing Cross Hospital Campus of Imperial College, London, UK. BCCA, Victoria, BC, Canada. ${ }^{3}$ Imprinting and Cancer Group, Cancer Epigenetic and Biology Program (PEBC), Bellvitge Biomedical Research Institute (IDIBELL), L'Hospitalet de Llobregat, Barcelona, Spain. ${ }^{4}$ Department of Medical Genetics, University of British Columbia, Vancouver, BC, Canada. ${ }^{5}$ State Key Lab of Molecular Oncology, Laboratory of Cell and Molecular Biology, National Cancer Center, Beijing, China. ${ }^{6}$ Department of Surgery and Cancer, Imperial College, London, UK.

Received: 26 February 2019 Accepted: 4 July 2019

Published online: 29 July 2019

\section{References}

1. Seckl MJ, Sebire NJ, Berkowitz RS. Gestational trophoblastic disease. Lancet. 2010;376:717-29.

2. Bagshawe KD, Dent J, Webb J. Hydatidiform mole in England and Wales in 1973-1983. Lancet. 1986;2(8508):673-7.

3. Hertz R, Li MC, Spencer DB. Effect of methotrexate therapy upon choriocarcinoma and chorioadenoma. Proc Soc Exp Biol Med. 1956;93:361-6.

4. Alifrangis $C$, Agarwal $R$, Short $D$, et al. EMA/CO for high-risk gestational trophoblastic neoplasia: good outcomes with induction low-dose etoposide-cisplatin and genetic analysis. J Clin Oncol. 2013;31:280-6.

5. Fearon ER, Vogelstein B. A genetic model for colorectal tumourigenesis. Cell. 1990;61:759-67.

6. Savage P. Clinical observations on chemotherapy curable malignancies: unique genetic events, frozen development and enduring apoptotic potential. BMC Cancer. 2015;15:11.

7. Jiao L, Ghorani E, Sebire NJ, Seckl MJ. Intraplacental choriocarcinoma: systematic review and management guidance. Gynecol Oncol. 2016;141:624-31.

8. Fisher RA, Kaur B. Molecular genotyping in the diagnosis of trophoblastic tumours. Diag Histopath. 2019;25:66-76.

9. Hernandez Mora JR, Tayama C, Sánchez-Delgado M, et al. Characterization of parent-of-origin methylation using the Illumina Infinium MethylationEPIC array platform. Epigenomics. 2018;10:941-54.

10. Monk D, Morales J, den Dunnen JT, et al. Nomenclature group of the European network for human congenital imprinting disorders. Recommendations for a nomenclature system for reporting methylation aberrations in imprinted domains. Epigenetics. 2018;13:117-21.

11. Sanchez-Delgado M, Court F, Vidal E, et al. Human oocyte-derived methylation differences persist in the placenta revealing widespread transient imprinting. PLoS Genet. 2016;12:e100642.

12. Martin-Trujillo A, Vidal E, Monteagudo-Sánchez A, et al. Copy number rather than epigenetic alterations are the major dictator of imprinted methylation in tumors. Nat Commun. 2017;8:467.

13. Monteagudo-Sánchez A, Sánchez-Delgado M, Mora JRH, et al. Differences in expression rather than methylation at placenta-specific imprinted loci is associated with intrauterine growth restriction. Clin Epigenetics. 2019;11:35.

14. Bibikova M, Le J, Barnes B, Saedinia-Melnyk S, Zhou L, Shen R, Gunderson KL. Genome-wide DNA methylation profiling using Infinium ${ }^{\circledR}$ assay. Epigenomics. 2009;1:177-200.

15. Novakovic B, Saffery R. Placental pseudo-malignancy from a DNA methylation perspective: unanswered questions and future directions. Front Genet. 2013:4:285

16. Kobayashi H, Sakurai T, Miura F, et al. High-resolution DNA methylome analysis of primordial germ cells identifies gender-specific reprogramming in mice. Genome Res. 2013;23:616-27. 
17. Hughes $L A$, Melotte $V$, de Schrijver J, et al. The CpG island methylator phenotype: what's in a name? Cancer Res. 2013;73:5858-68.

18. Xing $\mathrm{D}$, Zheng G, Pallavajjala A, et al. Lineage-specific alterations in gynecologic neoplasms with choriocarcinomatous differentiation: implications for origin and therapeutics. Clin Cancer Res. 2019; (In press).

19. Horowitz NS, Mirkovic J, Garcia E, et al. Targeted genomic profiling and PD-L1 expression in epithelioid trophoblastic tumours and placental site trophoblastic tumours. ISSTD meeting presentation. 2017.

20. Andersson AK, Ma J, Wang J, et al. St. Jude Children's Research Hospital-Washington University pediatric cancer genome project. The landscape of somatic mutations in infant MLL-rearranged acute lymphoblastic leukemias. Nat Genet. 2015;47:330-7.

21. Lee RS, Stewart C, Carter SL, et al. A remarkably simple genome underlies highly malignant pediatric rhabdoid cancers. J Clin Invest. 2012;122:2983-8.

22. Mack SC, Witt H, Piro RM, et al. Epigenomic alterations define lethal CIMPpositive ependymomas of infancy. Nature. 2014;506:445-50.

23. Mao TL, Kurman RJ, Huang CC, Lin MC, Shih IEM. Immunohistochemistry of choriocarcinoma: an aid in differential diagnosis and in elucidating pathogenesis. Am J Surg Pathol. 2007;31:1726-32.

24. Stovall TG, Ling FW, Gray LA. Single-dose methotrexate for treatment of ectopic pregnancy. Obstet Gynecol. 1991;77:754-7.

25. Novakovic B, Yuen RK, Gordon L, et al. Evidence for widespread changes in promoter methylation profile in human placenta in response to increasing gestational age and environmental/stochastic factors. BMC Genomics. 2011; 12:529.

26. Robinson WP, Price EM. The human placental methylome. Cold Spring Harb Perspect Med. 2015;5:a023044.

27. Lim YC, Li J, Ni Y, et al. A complex association between DNA methylation and gene expression in human placenta at first and third trimesters. PLoS One. 2017;12:e0181155.

28. Nordor AV, Nehar-Belaid D, Richon S, et al. The early pregnancy placenta foreshadows DNA methylation alterations of solid tumors. Epigenetics. 2017: 12:793-803.

29. Cao L, Wang $S$, Zhang $Y$, et al. Zinc-finger protein 471 suppresses gastric cancer through transcriptionally repressing downstream oncogenic PLS3 and TFAP2A. Oncogene. 2018;37:3601-16.

30. Zhang J, Zheng Z, Zheng J, et al. Epigenetic-mediated downregulation of zinc finger protein 671 (ZNF671) predicts poor prognosis in multiple solid tumors. Front Oncol. 2019;9:342.

31. Küppers R, Klein U, Hansmann ML, Rajewsky K. Cellular origin of human B-cell lymphomas. N Engl J Med. 1999;341:1520-9.

32. Creinin MD, Vittinghoff E, Keder L, Darney PD, Tiller G. Methotrexate and misoprostol for early abortion: a multicenter trial. I. Safety and efficacy. Contraception. 1996:53:321-7.

33. Bouffet E, Foreman N. Chemotherapy for intracranial ependymomas. Childs Nerv Syst. 1999;15:563-70.

\section{Publisher's Note}

Springer Nature remains neutral with regard to jurisdictional claims in published maps and institutional affiliations.

Ready to submit your research? Choose BMC and benefit from:

- fast, convenient online submission

- thorough peer review by experienced researchers in your field

- rapid publication on acceptance

- support for research data, including large and complex data types

- gold Open Access which fosters wider collaboration and increased citations

- maximum visibility for your research: over $100 \mathrm{M}$ website views per year

At $\mathrm{BMC}$, research is always in progress.

Learn more biomedcentral.com/submissions 